\title{
Probe Summary Method
}

National Cancer Institute

\section{Source}

National Cancer Institute. Probe Summary Method. NCI Thesaurus. Code C64221.

Method for summarizing expression values of all probes in a probe set. Defined in the documents available at http://bioconductor.org/packages/1.9/bioc/html/affy.html 